\title{
Neckteeth formation in two species of the Daphnia curvirostris complex (Crustacea: Cladocera)
}

\author{
Petr Jan JURAČKA*, Christian LAFORSCH ${ }^{1)}$, Adam PETRUSEK \\ Charles University in Prague, Faculty of Science, Department of Ecology, Viničná 7, CZ-12844 Prague, Czech Republic \\ ${ }^{1)}$ Ludwig-Maximilians-University Munich, Department of Biology II and GeoBio Center, Großhaderner Str. 2, D-82152 \\ Planegg-Martinsried, Germany \\ *e-mail corresponding author: juracka@natur.cuni.cz
}

\begin{abstract}
Cladocerans of the genus Daphnia show different morphological adaptations against invertebrate predation. Among those, the formation of neckteeth has attracted substantial attention. Morphotypes exhibiting neckteeth better resist predation from larvae of phantom midges Chaoborus (Diptera). These morphological structures are known from several species of the Daphnia longispina and $\mathrm{D}$. pulex complexes; recently they have also been reported in the $\mathrm{D}$. curvirostris complex, within which they are well documented from the Far East species D. sinevi and from Central European D. hrbaceki. Much scarcer are indications of the formation of these structures in the widespread species D. curvirostris. Careful inspection of samples from pools with Chaoborus larvae nevertheless revealed that a small necktooth in the first few instars of D. curvirostris is not uncommon, but probably has been mostly overlooked in the past. Occasionally, even adult D. curvirostris males may carry this feature. We provide documentation, particularly by scanning electron micrographs, of neckteeth in field-collected D. curvirostris, and in juvenile individuals of its sister species $\mathrm{D}$. hrbaceki. In addition, we tested the response of three clones each of $\mathrm{D}$. curvirostris and $\mathrm{D}$. hrbaceki to Chaoborus kairomones in laboratory experiments. Two clones of the former species and all three of the latter responded to this predator cue with neckteeth formation. First-instar juveniles of D. hrbaceki also occasionally carried neckteeth in control treatments without Chaoborus kairomones, but second and third instars did not. We also observed strong interclonal variation in neonate length in the presence of kairomones in this species. We provide a summary table listing all Daphnia species presently known to exhibit neckteeth, and propose that the ability to form these structures may be more widespread among common Daphnia species than previously assumed.
\end{abstract}

Key words: Daphnia hrbaceki, Chaoborus, interclonal variability, inducible defences, neckteeth, predation

\section{INTRODUCTION}

Inducible morphological defences are among the most interesting antipredator adaptations, as they are often very conspicuous traits. They have been documented in most groups of organisms, ranging from bacteria to vertebrates (Tollrian \& Harvell 1999). In aquatic environments, prey responses are often initiated by detecting predator kairomones, i.e., infochemicals associated with a particular predator (Dicke \& Sabelis 1988). In cladocerans of the genus Daphnia, phenotypic plasticity in antipredator defensive traits has been frequently studied. Various Daphnia species show striking protective morphological structures, such as helmets of various shapes in D. cucullata Sars, 1862 (Tollrian 1990) or D. longicephala Hebert, 1977 (Grant \& Bayly 1981), sharp spines in D. lumholtzi Sars, 1885 (Sorensen \& Sterner 1992; Tollrian 1994; Dzialowski et al. 2003), or spiny head lobes called the "crown of thorns" in the D. atkinsoni complex (Laforsch et al. 2009; Petrusek et al. 2009). Other morphological antipredator defences are much less obvious. In the presence of predatory phantom-midge (Chaoborus) larvae, a number of Daphnia species form neckteeth (Tab. 1), characteristic small spines on the dorsal part of their cara- pace. Although it had been shown that neckteeth efficiently increase the resistance of Daphnia to Chaoborus predation (Havel \& Dodson 1984; Repka et al. 1995), the mechanism of this protective effect remained unclear. Laforsch et al. (2004) nevertheless showed that the phenotypic changes accompanying neckteeth formation are much more complex, and involve not only superficially visible structures but also substantial strengthening of the carapace.

Neckteeth can be formed by various species of the subgenus Daphnia (sensu Johnson, 1952; i.e., including both $D$. longispina and $D$. pulex groups), especially in juvenile individuals (Colbourne et al. 1997; Kotov et al. 2006). The presence of neckteeth also recently received attention in the D. curvirostris complex, shown to contain several lineages in the Palaearctic region (Ishida et al. 2006; Kotov et al. 2006; Juračka et al. 2010). Species of this complex often live in small fishless pools where invertebrate predation is usually strong (Arnott \& Vanni 1993) and Chaoborus larvae are common (e.g., Kvam \& Kleiven 1995; Sell 2006). Despite this, an observation of neckteeth in a member of the D. curvirostris complex was pointed out in the literature only recently, for D. sinevi Kotov, Ishida \& Taylor, 2006, a species newly described from the Russian Far East (Kotov et al. 2006). This discovery provided additional 
Tab. 1. List of Daphnia lineages known to produce neckteeth, arranged according to their phylogenetic relationships. Species complexes are labelled according to Adamowicz et al. (2009), their phylogenies are provided in Adamowicz et al. (2009) and Juračka et al. (2010). Nomenclature of D. longispina follows Petrusek et al. (2008). Nomenclature of the D. pulex complex is not resolved (see, e.g., Mergeay et al. 2008); the lineage indicated as FLO9 was named D. arenata in Hebert (1995) and several subsequent publications but has never been formally described; different lineages are labelled $D$. pulicaria and $D$. pulex in the Old and the New World.

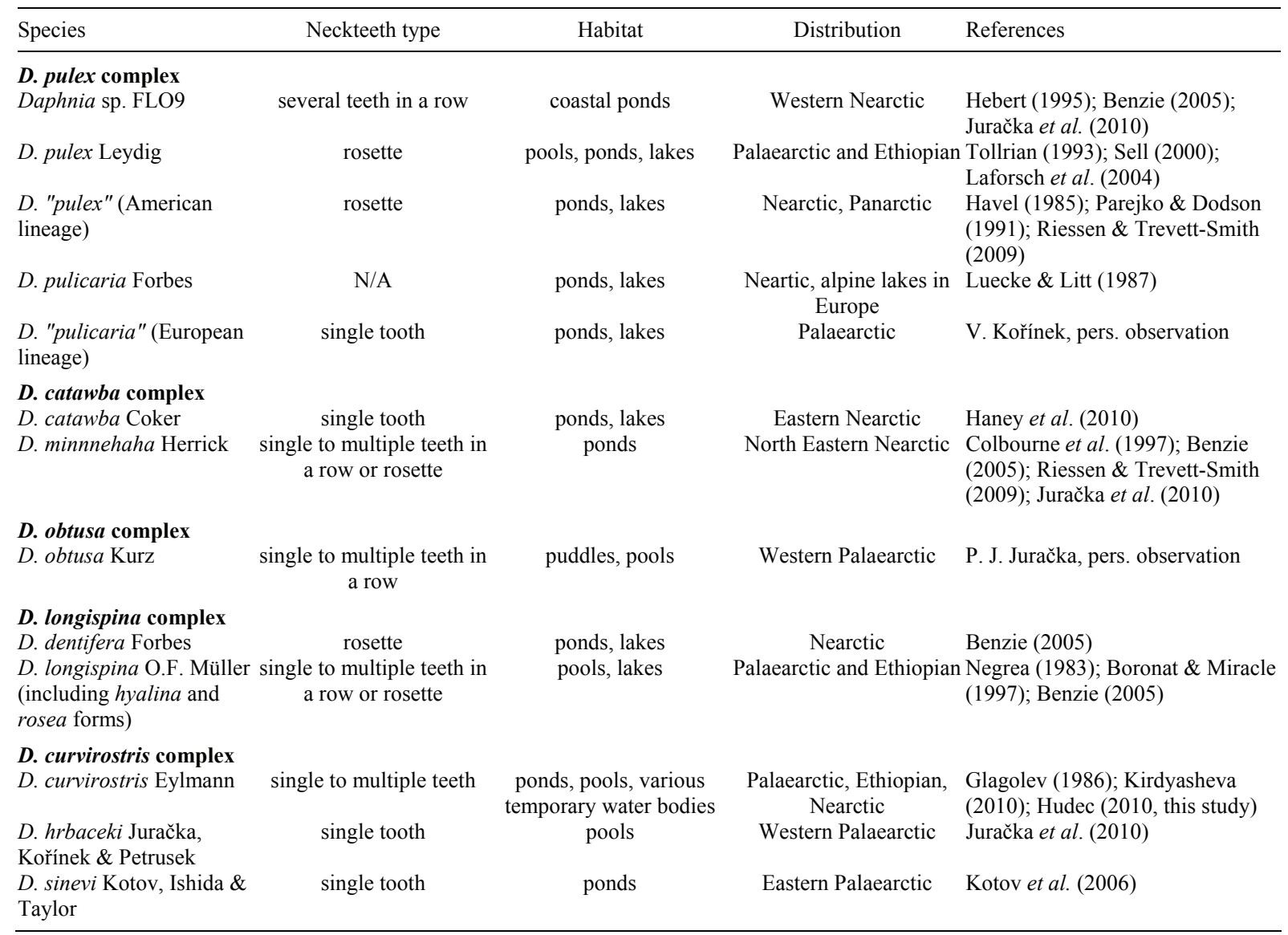

support for the conclusions of Colbourne et al. (1997) that neckteeth in Daphnia originated several times independently.

Another species of the D. curvirostris complex recently described from Central Europe, Daphnia hrbaceki Juračka, Kořínek \& Petrusek, 2010, exhibits this protective structure as well (Juračka et al. 2010). Juračka et al. (2010) observed neckteeth in both male and female juveniles and even in adults in the $D$. hrbaceki type locality, which was inhabited by Chaoborus larvae. In some natural populations, adults carrying neckteeth had a conspicuous hump-shaped dorsal body outline, presumably a phenotype accompanying the formation of inducible antipredator structures under certain environmental conditions. Similar forms are known from the North American species D. minnehaha Herrick, 1884 (Hebert 1995). D. hrbaceki escaped recognition and formal description for a long time, although its hump-shaped phenotypes strikingly differ from other European Daphnia species. Apparently, this is due to the fact that such morphs occur only under specific environmental conditions: while observ- ing the population at the species' type locality for several years, we noted that hump-shaped phenotypes slowly disappeared, despite the continuing presence of Chaoborus larvae (Juračka et al. 2010).

Even the most widespread member of the species complex, Daphnia curvirostris Eylmann, 1887, which has been known for more than a century, may apparently form neckteeth. However, this has been largely overlooked. When comparing D. hrbaceki to D. curvirostris to elucidate species-specific traits, we observed a single necktooth in juveniles and even adult males in some Central European populations of the latter species; we therefore searched for evidence for this feature in the available literature. To our knowledge, the only drawings of $D$. curvirostris with one necktooth have been given by Glagolev (1986) and Kirdyasheva (2010) from a Russian population. Additionally, Matile (1890) provided documentation of neckteeth formation in adult Daphnia specimens of a taxon described by him as $D$. dentata, which might belong to the $D$. curvirostris complex, from the vicinity of Moscow. Apart from formation of a single neckteeth in D. curvirostris, Hudec 
Tab. 2. Material analyzed morphologically in this study. Abbreviations of collector names: AGK: A.G. Kirdyasheva, DV: D. Vondrák, PJJ: P.J. Juračka, VK: V. Kořínek, VKr: V. Kraslová. All localities except Borok (Russia) are in the Czech Republic. Chaoborus was identified to species level if material was available; otherwise its presence is noted. NA indicates a pre-sorted sample where presence of this predator could not be evaluated.

\begin{tabular}{|c|c|c|c|c|c|}
\hline Locality & Coordinates & Locality type & Date & Chaoborus & Collector \\
\hline \multicolumn{6}{|l|}{ Daphnia curvirostris } \\
\hline Tupadly & $\begin{array}{l}\text { N 50²6'16" } \\
\text { E } 14^{\circ} 28^{\prime} 20^{\prime \prime}\end{array}$ & experimental pools & $\begin{array}{c}23 \text { October } 2007, \\
6 \text { October } 2008\end{array}$ & $\begin{array}{l}\text { C. crystallinus } \\
\text { C. obscuripes }\end{array}$ & DV \\
\hline $\begin{array}{l}\text { Vrbno, } \\
\text { near Smyslov pond }\end{array}$ & $\begin{array}{l}\text { N } 49^{\circ} 25^{\prime} 07^{\prime \prime} \\
\text { E } 13^{\circ} 48^{\prime} 10^{\prime \prime}\end{array}$ & temporary pool & 4 June 2010 & present & VK \\
\hline $\begin{array}{l}\text { Tchořovice, } \\
\text { near Radov pond }\end{array}$ & $\begin{array}{l}\text { N } 49^{\circ} 25^{\prime} 17^{\prime \prime} \\
\text { E } 13^{\circ} 49^{\prime} 22^{\prime \prime}\end{array}$ & forest pool & May 2010 & present & VK \\
\hline Tvrdonice & $\begin{array}{l}\text { N } 48^{\circ} 44^{\prime} 54^{\prime \prime} \\
\text { E } 17^{\circ} 01^{\prime} 25^{\prime \prime}\end{array}$ & temporary pool & 15 April 2008 & C. pallidus & $\mathrm{VKr}$ \\
\hline Borok (Russia) & $\begin{array}{l}\text { N } 58^{\circ} 03^{\prime} \\
\text { E } 38^{\circ} 13^{\prime}\end{array}$ & temporary puddles & 11 June 2004 & present & AGK \\
\hline \multicolumn{6}{|l|}{ Daphnia hrbaceki } \\
\hline $\begin{array}{l}\text { Nosálov } \\
\text { (type locality) }\end{array}$ & $\begin{array}{l}\text { N } 50^{\circ} 28^{\prime} 54^{\prime \prime} \\
\text { E } 14^{\circ} 41^{\prime} 10^{\prime \prime}\end{array}$ & pool & $\begin{array}{c}7 \text { samples } \\
\text { between May } \\
2005 \text { and } \\
\text { November } 2007\end{array}$ & C. crystallinus & PJJ \\
\hline Nosálov & 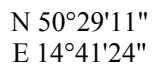 & pool & $\begin{array}{l}10 \text { November } \\
2006\end{array}$ & $\begin{array}{l}\text { C. crystallinus } \\
\text { C. flavicans }\end{array}$ & PJJ \\
\hline Drásov & $\begin{array}{l}\text { N 4941'37" } \\
\text { E } 14^{\circ} 06^{\prime} 19^{\prime \prime}\end{array}$ & temporary pool & 3 July 1995 & NA & VK \\
\hline \multicolumn{6}{|l|}{ Daphnia obtusa } \\
\hline Nosálov & $\begin{array}{l}\text { N } 50^{\circ} 29^{\prime} 11^{\prime \prime} \\
\text { E } 14^{\circ} 41^{\prime} 24^{\prime \prime}\end{array}$ & pool & $\begin{array}{c}4 \text { July } 2005, \\
13 \text { October } 2005\end{array}$ & C. crystallinus & PJJ \\
\hline \multicolumn{6}{|c|}{ D. "pulicaria" (European lineage) } \\
\hline Pole & $\begin{array}{l}\text { N } 49^{\circ} 25^{\prime} 23^{\prime \prime} \\
\text { E } 13^{\circ} 48^{\prime} 03^{\prime \prime}\end{array}$ & pool & 30 July 2010 & present & VK \\
\hline
\end{tabular}

(2010) documented a juvenile female ascribed to this species with multiple neckteeth from a Slovakian population, and Kirdyasheva (2010) reported that some juveniles from one of the Russian populations also carried three or more neckteeth. Several independent observations therefore confirm that $D$. curvirostris is able to form neckteeth; unfortunately, the above-cited works are mostly difficult to access.

The present study has two aims: 1) to provide light and scanning electron microscopy documentation of neckteeth in D. curvirostris, and compare them with those of its sister species $D$. hrbaceki; 2) to experimentally test whether neckteeth formation in both of these European members of the D. curvirostris complex can be induced by Chaoborus kairomones under laboratory conditions. Neckteeth induction has been successfully demonstrated in laboratory experiments with other Daphnia species (e.g., Havel \& Dodson 1987; Tollrian 1995; Sell 2000; Riessen \& Trevett-Smith 2009); we therefore hypothesized that both species would be responsive to Chaoborus cues.

\section{METHODS}

\subsection{Material examined}

The studied populations of Daphnia, particularly $D$. curvirostris and D. hrbaceki, used for neckteeth documentation and for laboratory experiments, are listed in table 2. If present in the samples, Chaoborus was identi- fied to species level according to Rozkošný et al. (1980).

For the first laboratory experiment, each species was represented by three different clones, distinguishable from each other by alleles at seven microsatellite loci (described in Brede et al. 2006): Dp281NB, DaB17/17, SwiD14, Dgm105, Dgm112, SwiD4, and SwiD18 (A. Thielsch, unpublished data). Two of those clones per species, together with a clone of Daphnia pulex Leydig, 1860 known to be well responsive to predator cues, were used in the second experiment. The D. pulex clone was included as a control for neckteeth formation; it was provided by Ralph Tollrian and has been cultured in the laboratory for several years.

\subsection{Documentation of neckteeth from field samples}

To document neckteeth from natural populations, we used both light and scanning electron microscopy (SEM). Photographs were taken by a Nikon D300 digital camera attached to an Olympus BX51TF optical microscope. A selected specimen was photographed 10 times with different depths of focus, and the resulting image was merged to gain extended depth of field with Helicon Focus 5.1.2. and Adobe Photoshop CS3 software.

Specimens used for SEM were dehydrated in a graded acetone series and then dried with organic volatile matter hexamethyldisalazane (Laforsch \& Tollrian 
2000). Dehydrated specimens were gold-coated in a BAL-TEC Sputter Coater SCD 050 for 5-7 minutes in argon plasma at $10^{-1}$ millibar vacuum. Then, they were imaged with a JEOL JSM-6380 LV scanning electron microscope.

\subsection{Experimental design}

We used three clones each of $D$. curvirostris and $D$. hrbaceki, sampled in late August 2006, to test their response to Chaoborus kairomones. D. curvirostris clones originated from shallow temporary pools near

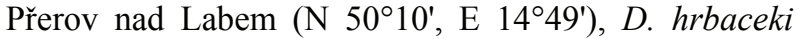
from its type locality near Nosálov (see Tab. 2). The animals were reared in the laboratory under constant conditions $\left(20^{\circ} \mathrm{C} \pm 0.5,16\right.$ hours of light per day) in artificial medium (according to Jeschke \& Tollrian 2000); local groundwater from Planegg-Martinsried was used instead of tap water. Daphnids were fed daily with Scenedesmus obliquus $\left(1.5 \mathrm{mg}\right.$ carbon $\left.\mathrm{L}^{-1}\right)$.

For the first experiment, we randomly selected six juvenile females of each clone and placed them into separate beakers (volume $1.5 \mathrm{~L}$ ). Into each of these beakers, we put a small plastic cage with the bottom made from a $200 \mu \mathrm{m}$ mesh, allowing the flow of infochemicals but not physical contact with the predator. In three beakers, the cage contained five specimens of the $4^{\text {th }}$ larval instar of Chaoborus crystallinus. The other three beakers containing daphnids of each clone served as control treatments without the predator presence. Chaoborus were fed with Daphnia neonates of the same clone as in the respective beaker to maximize the expression of morphological defences, as predators consuming conspecific prey are known to increase the formation of inducible defences (Stabell et al. 2003; Laforsch et al. 2006). To ensure sufficient mixing of predator kairomones and prey alarm substances with the culture medium, each cage with Chaoborus larvae was raised almost out of the medium and lowered back down twice a day. The medium in each beaker was changed with every reproductive event.

Daphnia individuals with which the experiment started (the "mother generation") were exposed to the predator cues to take maternal effects into account (Agrawal et al. 1999). We then used individuals from the third clutches of these females to evaluate the response of the next-generation juveniles to predator kairomones (neonates of the first and second clutch were removed and used as feed for Chaoborus). The third clutch neonates were counted, individually photographed to measure body and spine length (see below), and checked for the presence of neckteeth. Immediately afterwards, we randomly selected five individuals from the clutch (or less in cases of smaller clutches) and transferred them to separate $0.1 \mathrm{~L}$ beakers (the smaller flask volume was used due to space limitations) to follow the life history and morphological changes of each daphnid individually. The media were changed twice a day in each beaker. In Chaoborus treatments, the beakers contained culture medium with predator-conditioned water prepared as described for the mother generation (see above). The control medium contained only algal food but no predator or prey infochemicals. We took a second measurement of morphometric parameters of each individual Daphnia at the age of first reproduction, and evaluated the number of offspring in their first clutch.

To compare neckteeth formation among first three juvenile instars, we performed a second experiment using two clones of each species tested in the first experiment (D. hrbaceki clones 2 and 3, and D. curvirostris clones 1 and 3 ). In addition, we also exposed a clone of D. pulex to Chaoborus kairomones to test for the efficiency of the predator cue, as this species is known to exhibit distinct neckteeth in response to Chaoborus (e.g., Tollrian 1995). The experimental design was similar to our first experiment, with the exception that we did not transfer the juveniles of the third brood of preconditioned mothers separately into small beakers but kept them in the original vessel to constantly expose the animals to predator cues. In addition, we used ten Chaoborus larvae per litre to increase the concentration of predator cues. We randomly selected 20 individuals (if available) in three consecutive days to collect animals of the first three instars. We checked for presence or absence of neckteeth in these instars under a Leica M10 stereomicroscope.

\subsection{Measurements and statistical analyses}

Photographs of each measured individual from the first experiment were taken by an Olympus ALTRA20 digital camera mounted on a Leica M10 stereomicroscope. Subsequently, we measured two morphometric parameters in the software Olympus cell ${ }^{\wedge} \mathrm{P}$ : body length (defined as the length between the upper edge of the compound eye to the base of the tail spine) and tail spine length (a straight line between the base of the tail spine and its top). Occasional juvenile individuals that were substantially larger than the others were removed from the dataset, as we suspected them of already being in the second instar. We also measured the body length of randomly selected neckteeth-carrying individuals from one population of each species (D. curvirostris: Tvrdonice, 15 April 2008; D. hrbaceki: type locality near Nosálov, 17 August 2006), to evaluate their size distributions and thus check whether neckteeth are present in different instars.

We used Pearson's Chi-square test to compare ratios of induced (i.e., with neckteeth) and uninduced specimens within each species in both experiments. Since we used 3 tests in the second experiment, we applied consequent manual Hochberg's p-value adjustment (Benjamini \& Hochberg 1995) for multiple testing. The morphometric parameters were compared between individuals in Chaoborus and control treat- 


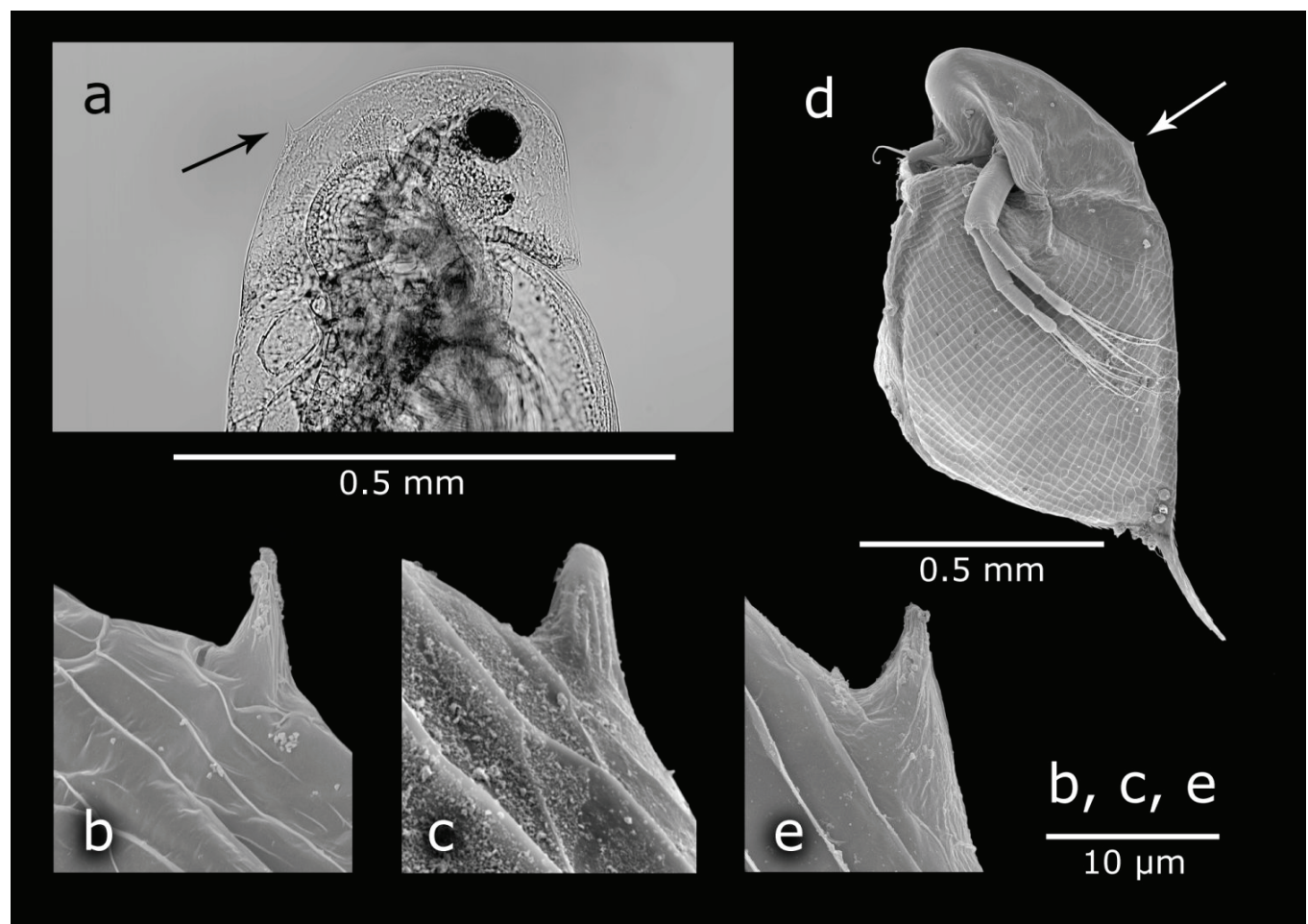

Fig. 1. Neckteeth in Czech populations of the Daphnia curvirostris complex. D. curvirostris from Tupadly: head (A) and necktooth detail (B) of juvenile females; an adult male in lateral view (D); detail of an adult male necktooth (E). D. hrbaceki: necktooth of a juvenile female from Nosálov (C). More figures of $D$. hrbaceki with neckteeth are available in Juračka et al. (2010). Arrows indicate neckteeth.

ments by a series of non-parametric Wilcoxon's signedrank test with consequent manual Hochberg's $p$-value adjustment. As the sizes of different specimens within one clutch cannot be considered independent replicates, we averaged them for each clutch, and used a single value for the whole clutch. Wilcoxon's signed-rank test was also used for comparing the size of clutches from controls and Chaoborus treatments.

\section{RESULTS}

In the samples of Daphnia curvirostris originating from Czech and Russian pools with Chaoborus larvae, most juveniles carried a small $(5-10 \mu \mathrm{m})$ necktooth (Fig. $1 \mathrm{a}, \mathrm{b})$. A necktooth of approximately the same size was also occasionally retained in adult males (Fig. 1d, e), as seen in field samples from Tupadly, Czech Republic (but also documented from Borok, Russia; Kirdyasheva 2010). Neckteeth of juvenile D. hrbaceki (Fig. 1c) were of a similar morphology as those of D. curvirostris. In both species, neckteeth were carried by a wide size range of juveniles, clearly indicating that the structure is present in several juvenile instars: the size of measured neckteeth-carrying individuals ranged between $0.61 \mathrm{~mm}$ $1.26 \mathrm{~mm}$ in $D$. curvirostris from Tvrdonice, and between 0.52 and $1.04 \mathrm{~mm}$ in $D$. hrbaceki from its type locality.

Juveniles of both D. curvirostris and D. hrbaceki also formed neckteeth during the laboratory experiments (Tab. 3); these individuals did not differ phenotypically from those in the natural populations. In the first experiment, D. hrbaceki had a much stronger tendency to form these structures: in all three tested clones, all first-instar juveniles carried a necktooth in the treatment with Chaoborus kairomones. Interestingly, some firstinstar juveniles with neckteeth were also found in the control treatments; their proportion was nevertheless significantly lower than in the Chaoborus treatments. A small proportion of individuals from one of the three tested D. curvirostris clones also formed neckteeth; however, there was no significant difference between controls and Chaoborus treatments. In the second experiment, however, almost all specimens of all three instars of both species produced neckteeth in the presence of Chaoborus, while those not exposed to predator cues only formed these structures in the first instar in $D$. hrbaceki (Tab. 3). No specimen of D. hrbaceki with a hump-shaped carapace (as found in the wild) was observed in the laboratory experiments.

Differences in daphnid morphometric and life history traits measured in the first experiment were not consistent between the Chaoborus and control treatments, either between the two tested species or among clones within species. We did not observe any clear trends or significant differences in size at first reproduction, clutch size, or relative spine length. The neonate size, however, showed interesting patterns (Fig. 2). D. hrbaceki clones 1 and 3 formed significantly larger neonates in the presence of Chaoborus than in controls 
Tab. 3. Ratios of induced (with neckteeth) and uninduced neonates of Daphnia hrbaceki and $D$. curvirostris in the laboratory induction experiments. D. pulex served as a control for the efficiency of the predator cue in the second experiment. Significances of differences between Chaoborus and control treatments were tested by the Pearson's Chisquare test (adjusted $p$-values are given for the second experiment).

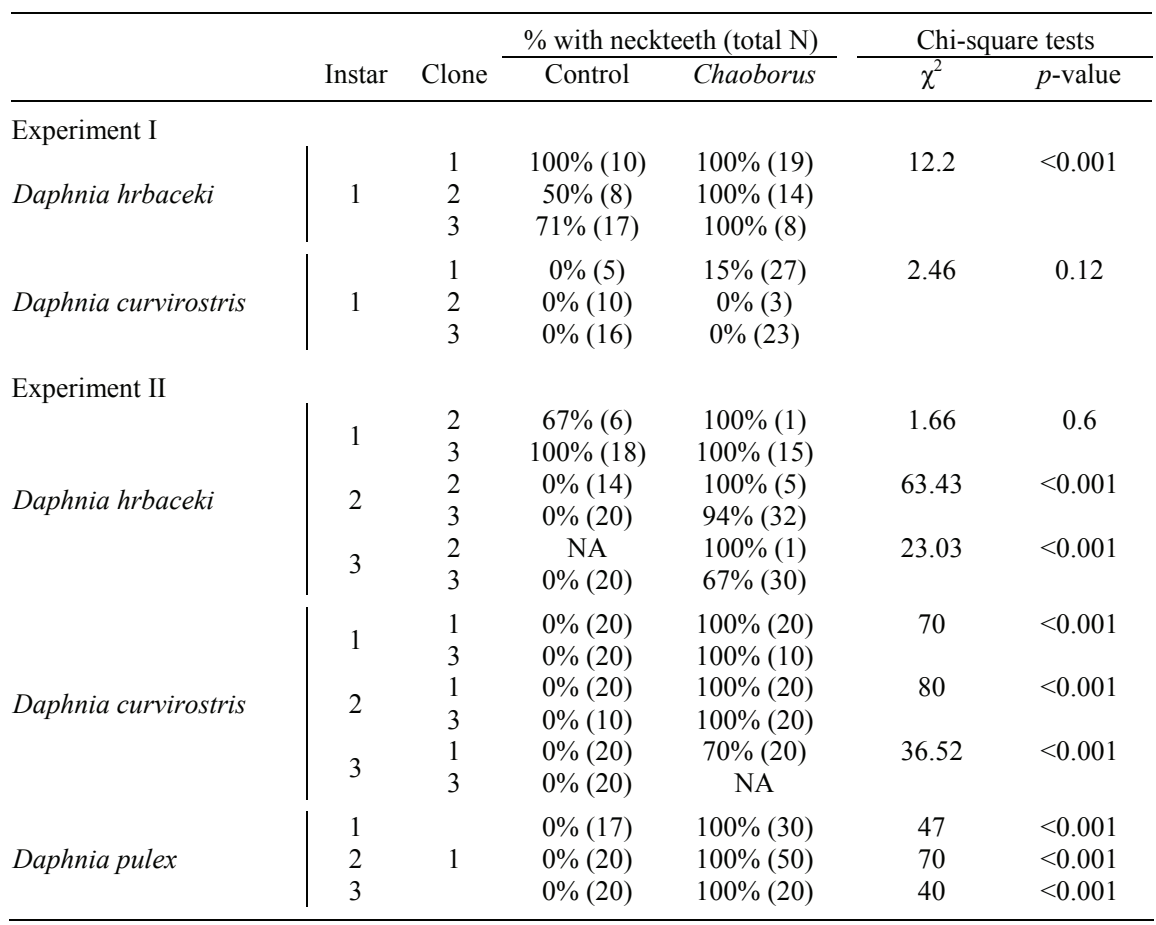
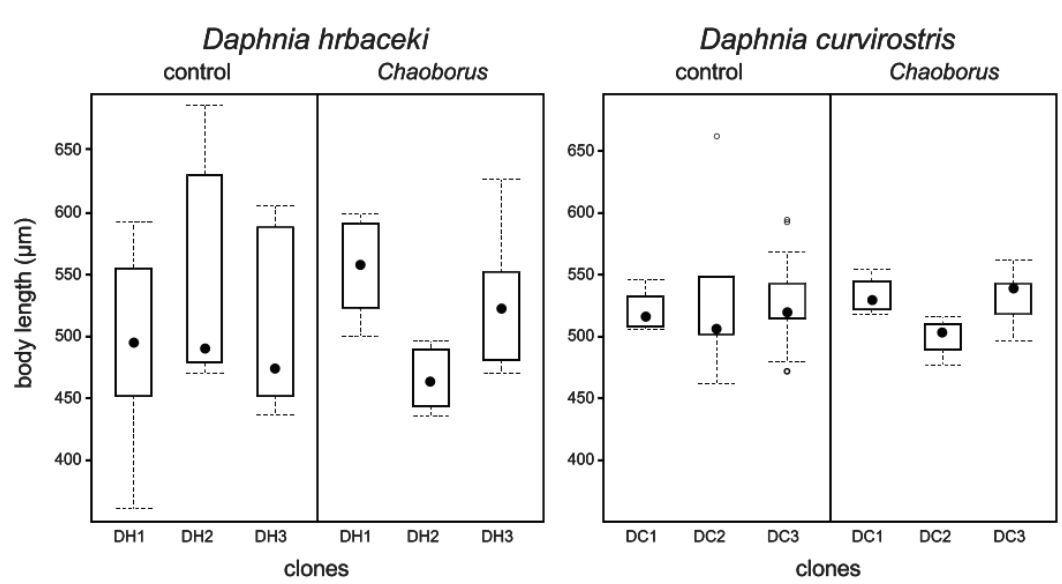

Fig. 2. Body length of Daphnia hrbaceki and Daphnia curvirostris third-clutch neonates in absence and presence of Chaoborus kairomones in the laboratory induction experiment. Median (dark circle), interquartile ranges (box) and non-outlier ranges (whiskers) are shown by the box-and-whisker plot; outliers are indicated by empty circles. D. hrbaceki clones 1 and 3 were significantly larger in the kairomone treatment than in the control, clone 2 was significantly smaller. In $D$. curvirostris, differences in neonate lengths from kairomone and control treatments were not significant.

(Wilcoxon's signed-rank tests with Hochberg's $p$-value adjustment; adjusted $p=0.036, \mathrm{~W}=0$ and $0.027, \mathrm{~W}=$ 0 , respectively); on the contrary, clone 2 neonates were significantly smaller under the same conditions (adjusted $p=0.027, \mathrm{~W}=58)$. D. curvirostris clones did not exhibit any difference in neonate size between treatments (adjusted $p>0.376, \mathrm{~W} \leq 7$ in all three comparisons). We also did not observe any trade-off between neonate size within the clutch and clutch size in either of the tested species.

\section{DISCUSSION}

Our study confirms that both studied European species of the Daphnia curvirostris complex are able to form neckteeth in the field as well as under laboratory conditions, and in several juvenile instars. However, we observed neckteeth formation in the $D$. hrbaceki first instar not only in the presence of Chaoborus kairomones but also in the treatments without predator cues. Similar observations are known from some lineages of 
the D. pulex complex, including European D. pulex Leydig, 1860 (Tollrian 1993) as well as the North American D. "pulex" (H. Riessen, personal communication); additionally, Kirdyasheva (2010) reported neckteeth in juvenile instars of $D$. curvirostris from a population where Chaoborus had not been observed. Spontaneous neckteeth development in neonates of these species may be explained by the fact that they occur in fishless habitats where invertebrate predation is often very strong. Hence, neckteeth development even in the absence or low concentration of Chaoborus cues may be a good start-up defence, as predation pressure may change rapidly.

Based on previous experimental work on other Daphnia species (Havel 1985; Tollrian 1993), we expected that our studied taxa should produce a higher ratio of neonates with neckteeth in Chaoborus treatments than in controls. The results were consistent with this hypothesis in both experiments (Tab. 3). In the first experiment, the trend was significant only for $D$. hrbaceki, in which all neonate individuals of all three tested clones carried neckteeth in the Chaoborus treatment. In the second experiment, the kairomone effect was much stronger, and differences between control and predator treatments were highly significant in almost all species and instars (with the exception of the $D$. hrbaceki first instar). The difference between the experiments may be explained by doubled kairomone concentration in the second one. This corresponds to results of previous studies reporting the influence of kairomone dose on the formation of protective traits (e.g., Tollrian 1993). In the first experiment that focused on neonates only, $D$. curvirostris formed neckteeth much less frequently than $D$. hrbaceki (only $15 \%$ of juveniles of a single clone in the Chaoborus treatment). This is in accordance with the infrequent field observations of $D$. curvirostris populations with neckteeth, and suggests that $D$. hrbaceki is more likely to respond with morphological defences under low kairomone concentrations.

In our experiments, individuals of both species showing neckteeth exclusively formed a single necktooth. We did not observe any rosette-like neckteeth formed by more dorsal spines, as documented in $D$. curvirostris by Hudec (2010) and Kirdyasheva (2010). Their field observations nevertheless suggest that the taxon is one of those Daphnia species that are plastic in their level of neckteeth expression (see Tab. 1).

In both experiments, we did not observe any humpshaped morphs. The failure to produce inducible defences as strong as those seen in the wild is common in laboratory experiments (Dodson 1988; Tollrian 1994; Laforsch \& Tollrian 2004; Tanner \& Branstrator 2006). In our case, this may be due to various reasons. It could be due to an incomplete or insufficiently intense inducing stimulus. Tanner \& Branstrator (2006) found a three-generation delay in D. mendotae Birge, 1918 producing a round helmet in reaction to the predatory cladoceran Leptodora; possibly, a dorsal hump in $D$. hrbaceki may only be formed in an experiment spanning several generations. Riessen and Young (2005) suppose that similar hump-shaped phenotypes in North American D. minnehaha are induced by the predator only under low-food conditions. This synergistic interaction would correspond to the field observations of $D$. hrbaceki from its type locality, a newly excavated pool. Hump-shaped morphs were common there during the first years of habitat existence, but disappeared two to three years later when the trophic status of the habitat substantially increased (Juračka et al. 2010). In addition, it has been shown that small scale turbulence evoked by the movement of predators can act synergistically with chemical cues to induce maximal trait responses (Tollrian \& Laforsch 2006). Hence, synergistic effects of kairomones and environmental conditions are well known within the Daphnia genus (e.g., Weber 2001; Weetman \& Atkinson 2002; Tollrian \& Laforsch 2006), and may also explain the absence of hump-shaped morphs in our experiments.

Daphnia are known to react to the presence of predators not only through morphological changes, but also by adaptive shifts in their life history (e.g., Schwartz 1984; Weber \& Declerck 1997; Boersma et al. 1998). Among the most common changes are alterations in the number and size of offspring through maternal effects, depending on the specific predators (Tollrian 1995; Agrawal et al. 1999). In the presence of predators preferring larger prey (particularly fish), some species tend to produce smaller neonates (Reede 1997; De Meester \& Weider 1999; Spaak et al. 2000; Mikulski 2001). On the other hand, the same prey species may follow the opposite strategy in the presence of predators which are gape-limited, including Chaoborus (Pastorok 1981). In this case, females exposed to predator kairomones usually tend to produce large neonates (Riessen \& Sprules 1990; Lüning 1992; Spitze 1992; Tollrian 1995; but for exception, see Spitze, 1992).

As both studied species occur in small fishless pools with frequent strong predation pressure by Chaoborus (Mura \& Brecciaroli 2003; Louette \& De Meester 2005; Juračka et al. 2010), a tendency to increase neonate size in the kairomone treatments could have been expected. However, although all three clones of D. hrbaceki reacted to Chaoborus cues with a significant change in neonate size, the direction of this change varied among the clones. Two clones produced significantly larger neonates, while the third one produced smaller ones (Fig. 2). Strong interclonal variability in the reaction to predator kairomones is well known from previous laboratory experiments in Daphnia (Parejko \& Dodson 1991; Weber \& Declerck 1997; Boersma et al. 1998), including opposite reactions within one species (Spitze 1992; Boersma et al. 1998; Pauwels et al. 2005).

Based on field observations, we suppose that neckteeth are induced relatively often as a defence against 
Chaoborus predation, probably also by other species of the subgenus Daphnia in which this feature is not known. It is generally assumed that neckteeth in Daphnia originated multiple times independently, and this hypothesis has been suggested by several authors. Colbourne et al. (1997) and Kotov et al. (2006) came to this conclusion because neckteeth had been documented only sporadically in distinct Daphnia species complexes. The potential independent origin of neckteeth in $D$. longispina and D. pulex groups (treated as distinct subgenera) was also discussed by Beaton and Hebert (1997) in their study of the cellular basis of Daphnia head morphology. Representatives of the two groups differed in the number of polyploid cells in the muscle attachment region, which might be responsible for neckteeth formation. Colbourne et al. (1997) also claimed, in support of the multiple-origin hypothesis, that some Daphnia species living mostly in small turbid habitats without Chaoborus, e.g., North American members of the D. obtusa complex, do not produce neckteeth even in experiments with Chaoborus kairomones. However, Beaton \& Hebert (1997) proposed a potential for neckteeth formation in three species of that complex, although they lacked evidence of this ability from field samples or laboratory collections. This is in agreement with field observations from Europe: D. obtusa Kurz, 1874 (sensu stricto) does produce neckteeth in pools with high Chaoborus abundances (P.J. Juračka, personal observation).

The growing evidence that neckteeth are more common than previously assumed among various daphnids from both the pulex and longispina groups may also give some support to an alternative scenario of evolution of neckteeth defences. Ontogenetic mechanisms allowing neckteeth formation could be a plesiomorphic character, expressed only in taxa where selection by predators strongly favoured them. This is further supported by the fact that some species apparently exhibit different forms of neckteeth (ranging from single to multiple arranged in a row or a rosette-like fashion). Further research into the genomic basis of neckteeth formation may reveal whether the different forms of neckteeth are homologous in unrelated Daphnia species or not.

\section{ACKNOWLEDGEMENTS}

We thank Jiři Hotový for sampling of D. curvirostris, Anne Thielsch for providing genetically distinguished experimental clones, David Hardekopf for language corrections, and all people who helped with maintenance of the experiment, especially Mechthild Kredler, Justyna Wolinska, Max S. Rabus and Zuzana Lulayová. Vladimír Kořínek, Alexey A. Kotov, Veronika Kraslová and Dan Vondrák provided field samples of D. curvirostris. This work as part of the STRESSFLEA project of the European Science Foundation EUROCORES Programme EuroEEFG was sup- ported from funds of the Czech Science Foundation (project EEF/10/E022) and German Research Foundation - DFG (LA 2159/6-1). Further funding was provided by the Grant Agency of the Charles University (5325/2010), the Czech Science Foundation (P506/10/P167) and the Czech Ministry of Education (MSM0021620828). We thank Alexey A. Kotov and Howard P. Riessen for reviews that improved a previous version of the manuscript.

\section{REFERENCES}

Adamowicz, S.J., A. Petrusek, J.K. Colbourne, P.D.N. Hebert \& J.D.S. Witt. 2009. The scale of divergence: A phylogenetic appraisal of intercontinental allopatric speciation in a passively dispersed freshwater zooplankton genus. Mol. Phylogenet. Evol., 50: 423-436.

Agrawal, A.A., C. Laforsch \& R. Tollrian. 1999. Transgenerational induction of defenses in animals and plants. Nature, 401: 60-63.

Arnott, S.E. \& M.J. Vanni. 1993. Zooplankton assemblages in fishless bog lakes: influence of biotic and abiotic factors. Ecology, 74: 2361-2380.

Beaton, M.J. \& P.D.N. Hebert. 1997. The cellular basis of divergent head morphologies in Daphnia. Limnol. Oceanogr., 42: 346-356.

Benjamini, Y. \& Y. Hochberg. 1995. Controlling the false discovery rate: A practical and powerful approach to multiple testing. J. Roy. Stat. Soc. B Met., 57: 289-300.

Benzie, J.A.H. 2005. The genus Daphnia (including Daphniopsis) (Anomopoda: Daphniidae). Kenobi Productions, Ghent: 376 pp.

Boersma, M., P. Spaak \& L. De Meester. 1998. Predator-mediated plasticity in morphology, life history, and behavior of Daphnia: the uncoupling of responses. Am. Nat., 152: 237-248.

Boronat, M.D. \& M.R. Miracle. 1997. Size distribution of Daphnia longispina in the vertical profile. Hydrobiologia, 360: 187-196.

Brede, N., A. Thielsch, C. Sandrock, P. Spaak, B. Keller, B. Streit \& K. Schwenk. 2006. Microsatellite markers for European Daphnia. Mol. Ecol. Notes., 6: 536-539.

Colbourne, J.K., P.D.N. Hebert \& D.J. Taylor. 1997. Evolutionary origins of phenotypic plasticity in Daphnia. In: Givnish, T.J. \& K.J. Sytsma (Eds), Molecular evolution and adaptive radiation. Cambridge University Press: 163-188.

De Meester, L. \& L.J. Weider. 1999. Depth selection behavior, fish kairomones, and the life histories of Daphnia hyalina $\times$ galeata hybrid clones. Limnol. Oceanogr., 44: 1248-1258.

Dicke, M. \& M.W. Sabelis. 1988. Infochemical terminology: based on cost-benefit analysis rather than origin of compounds? Funct. Ecol., 2: 131-139.

Dodson, S.I. 1988. Cyclomorphosis in Daphnia galeata mendotae Birge and D. retrocurva Forbes as a predator-induced response. Freshwat. Biol., 19: 109-114.

Dzialowski, A.R., J.T. Lennon, W.J. O'Brien \& V.H. Smith. 2003. Predator-induced phenotypic plasticity in the exotic cladoceran Daphnia lumholtzi. Freshwat. Biol., 48: 15931602.

Glagolev, S.M. 1986. Morphology, systematics and geographic distribution of the cladoceran genus Daphnia from Eurasia. PhD thesis, AN Severtsov Institute of Evolutionary Morphology and Ecology of Animals, Academy of Sciences USSR (in Russian).

Grant, J.W.G. \& I.A.E. Bayly. 1981. Predator induction of crests in morphs of the Daphnia carinata King complex. Limnol. Oceanogr., 26: 201-218. 
Haney, J.F., M.A. Aliberti, A. Elisha, S. Allard, D.J. Bauer, W. Beagen, S.R. Bradt, B. Carlson, S.C. Carlson, U.M. Doan, J. Dufresne, W.T. Godkin, S. Greene, A. Kaplan, E. Maroni, S. Melillo, A.L. Murby, J.L. Smith (Nowak), B. Ortman, J.E. Quist, S. Reed, T. Rowin, M. Schmuck \& R.S. Stemberger. 2010. An Image-based Key to the Zooplankton of the Northeast, USA. Version 4.0 released 2010. University of New Hampshire Center for Freshwater Biology. <http://cfb.unh.edu/CFBKey/html/index.html> [Accessed on January 17, 2011].

Havel, J.E. 1985. Cyclomorphosis of Daphnia pulex spined morphs. Limnol. Oceanogr., 30: 853-861.

Havel, J.E. \& S.I. Dodson. 1984. Chaoborus predation on typical and spined morphs of Daphnia pulex: Behavioral observations. Limnol. Oceanogr., 29: 487-494.

Havel, J.E. \& S.I. Dodson. 1987. Reproductive costs of Chaoborus-induced polymorphism in Daphnia pulex. Hydrobiologia, 150: 273-281.

Hebert, P.D.N. (1995) The Daphnia of North America: an illustrated fauna. CD-ROM. University of Guelph, Guelph.

Hudec, I. 2010. Anomopoda, Ctenopoda, Haplopoda, Onychopoda (Crustacea: Branchiopoda). VEDA vydavatel'stvo SAV, Bratislava: 496 pp (in Slovak).

Ishida, S., A.A. Kotov \& D.J. Taylor. 2006. A new divergent lineage of Daphnia (Cladocera: Anomopoda) and its morphological and genetical differentiation from Daphnia curvirostris Eylmann, 1887. Zool. J. Linn. Soc.-Lond., 146: 385-405.

Jeschke, J.M. \& R. Tollrian. 2000. Density-dependent effects of prey defences. Oecologia, 123: 391-396.

Johnson, D.S. 1952. The British species of the genus Daphnia (Crustacea: Cladocera). Proc. Zool. Soc. London, 122: 435-462.

Juračka, P.J., V. Kořínek \& A. Petrusek. 2010. A new Central European species of the Daphnia curvirostris complex, Daphnia hrbaceki sp. nov. (Cladocera, Daphniidae). Zootaxa, 2718: 1-22.

Kirdyasheva, A.G. 2010. Novye dannye po morfologii Daphnia curvirostris Eylmann (Cladocera: Daphniidae) vo vremennych vodoemach basseina Rybinskogo vodokhranilischa. [New data on morphology of Daphnia curvirostris Eylmann (Cladocera: Daphniidae) in temporary waters of the basin of Rybinsk reservoir]. In: Ekologia $i$ morfologia bespozvonochnych kontinentalnych vod. Sbornik nauchnych rabot posvyaschennyj 100-letiu so dnia rozhdenia Philareta Dmitrievicha Mordukhai-Boltovskogo [Ecology and morphology of invertebrates of contitnetal waters. Collection of scientific works to celebrate 100 years since the birth of Philaret Dmitrievich Mordukhai-Boltovskoi]. Institute Biologii Vnutrennich Vod im. I. D. Papanina Rossijskoj Akademii Nauk [Institute for Biology of Inland Water RAS], Borok: pp. 165-179.

Kotov, A.A., S. Ishida \& D.J. Taylor. 2006. A new species in the Daphnia curvirostris (Crustacea: Cladocera) complex from the eastern Palearctic with molecular phylogenetic evidence for the independent origin of neckteeth. $J$. Plankton Res., 28: 1067-1079.

Kvam, O.V. \& O.T. Kleiven. 1995. Diel horizontal migration and swarm formation in Daphnia in response to Chaoborus. Hydrobiologia, 307: 177-184.

Laforsch, C. \& R. Tollrian. 2000. A new preparation technique of daphnids for Scanning Electron Microscopy using hexamethyldisilazane. Arch. Hydrobiol., 149: 587-596.

Laforsch, C. \& R. Tollrian. 2004. Inducible defenses in multipredator environments: cyclomorphosis in Daphnia cucullata. Ecology, 85: 2302-2311.

Laforsch, C., L. Beccara \& R. Tollrian. 2006. Inducible defenses: The relevance of chemical alarm cues in Daphnia. Limnol. Oceanogr., 51: 1466-1472.

Laforsch, C., W. Ngwa, W. Grill \& R. Tollrian. 2004. An acoustic microscopy technique reveals hidden morpho- logical defenses in Daphnia. Proc. Natl. Acad. Sci. USA, 101: 15911-15914.

Laforsch, C., A. Haas, N. Jung, K. Schwenk, R. Tollrian \& A. Petrusek. 2009. "Crown of thorns" of Daphnia: An exceptional inducible defense discovered by DNA barcoding. Commun. Integr. Biol., 2: 379-381.

Louette, G. \& L. De Meester. 2005. High dispersal capacity of cladoceran zooplankton in newly founded communities. Ecology, 86: 353-359.

Luecke, C. \& A.H. Litt. 1987. Effects of predation by Chaoborus flavicans on crustacean zooplankton of Lake Lenore, Washington. Freshwat. Biol., 18: 185-192.

Lüning, J. 1992. Phenotypic plasticity of Daphnia pulex in the presence of invertebrate predators: morphological and life history responses. Oecologia, 92: 383-390.

Matile, P. 1890. Die cladoceren der Umgegend von Moskau. Bull. Soc. imp. nat. Moscau, 1890(1): 1-67, pl. III-V.

Mergeay, J., X. Aguilera, S. Declerck, A. Petrusek, T. Huyse \& L. De Meester. 2008. The genetic legacy of polyploid Bolivian Daphnia: the tropical Andes as a source for the North and South American D. pulicaria complex. Mol. Ecol., 17: 1789-1800.

Mikulski, A. 2001. The presence of fish induces the quick release of offspring by Daphnia. Hydrobiologia, 442: 195-198.

Mura, G. \& B. Brecciaroli. 2003. The zooplankton crustacean of the temporary waterbodies of the Oasis of Palo (Rome, central Italy). Hydrobiologia, 495: 93-102.

Negrea, S. 1983. Cladocera. Academia Republicii Socialiste România, Bucuresti: 399 pp.

Parejko, K. \& S.I. Dodson. 1991. The evolutionary ecology of an antipredator reaction norm: Daphnia pulex and Chaoborus americanus. Evolution, 45: 1665-1674.

Pastorok, R.A. 1981. Prey vulnerability and size selection by Chaoborus larvae. Ecology, 62: 1311-1324.

Pauwels, K., R. Stoks \& L. De Meester. 2005. Coping with predator stress: interclonal differences in induction of heat-shock proteins in the water flea Daphnia magna. J. evol. Biol., 18: 867-872.

Petrusek, A., R. Tollrian, K. Schwenk, A. Haas \& C. Laforsch. 2009. A "crown of thorns" is an inducible defense that protects Daphnia against an ancient predator. Proc. Natl. Acad. Sci. USA, 106: 2248-2252.

Petrusek, A., A. Hobæk, J.P. Nilssen, M. Skage, M. Černý, N. Brede \& K. Schwenk. 2008. A taxonomic reappraisal of the European Daphnia longispina complex (Crustacea, Cladocera, Anomopoda). Zool. Scr., 37: 507-519.

Reede, T. 1997. Effects of neonate size and food concentration on the life history responses of a clone of the hybrid Daphnia hyalina $\times$ galeata to fish kairomones. Freshwat. Biol., 37: 389-396.

Repka, S., M. Walls \& M. Ketola. 1995. Neck spine protects Daphnia pulex from predation by Chaoborus, but individuals with longer tail spine are at a greater risk. $J$. Plankton Res., 17: 393-403.

Riessen, H.P. \& W.G. Sprules. 1990. Demographic costs of antipredator defenses in Daphnia pulex. Ecology, 71: 1536-1546.

Riessen, H.P. \& J.B. Trevett-Smith. 2009. Turning inducible defenses on and off: adaptive responses of Daphnia to a gape-limited predator. Ecology, 90: 3455-3469.

Riessen, H.P. \& J.D. Young. 2005. Daphnia defense strategies in fishless lakes and ponds: one size does not fit all. $J$. Plankton Res., 27: 531-544.

Rozkošný, R., J. Ježek, J. Knoz, J. Kramář, F. Krampl, F. Kubíček, J. Lellák, J. Mináŕ, P. Pokorný, J. Raušer, E. Sedlák, V. Špačková, J.M. Štusák, J. Zelený \& M. Zelinka. 1980. Klič vodních larev hmyzu [Identification key of aquatic insect larvae]. Československá akademie věd, Prague: $524 \mathrm{pp}$.

Sell, A.F. 2000. Morphological defenses induced in situ by the invertebrate predator Chaoborus: comparison of responses 
between Daphnia pulex and D. rosea. Oecologia, 125: 150-160.

Sell, A.F. 2006. A trophic cascade with Chaoborus: population dynamics of ex-ephippial generations of Daphnia. Arch. Hydrobiol., 167: 115-134.

Schwartz, S.S. 1984. Life history strategies in Daphnia: A review and predictions. Oikos, 42: 114-122.

Sorensen, K.H. \& R.W. Sterner. 1992. Extreme cyclomorphosis in Daphnia lumholtzi. Freshwat. Biol., 28: 257-262.

Spaak, P., J. Vanoverbeke \& M. Boersma. 2000. Predator-induced life-history changes and the coexistence of five taxa in a Daphnia species complex. Oikos, 89: 164-174.

Spitze, K. 1992. Predator-mediated plasticity of prey life history and morphology: Chaoborus americanus predation on Daphnia pulex. Am. Nat., 139: 229-247.

Stabell, O.B., F. Ogbebo \& R. Primicerio. 2003. Inducible defences in Daphnia depend on latent alarm signals from conspecific prey activated in predators. Chemical Senses, 28: 141-153.

Tanner, C.J. \& D.K. Branstrator. 2006. Generational and dualspecies exposures to invertebrate predators influence relative head size in Daphnia mendotae. J. Plankton Res., 28: 793-802.

Tollrian, R. 1990. Predator-induced helmet formation in Daphnia cucullata (Sars). Arch. Hydrobiol., 119: 191-196.

Tollrian, R. 1993. Neckteeth formation in Daphnia pulex as an example of continuous phenotypic plasticity - morpho- logical effects of Chaoborus kairomone concentration and their quantification. J. Plankton Res., 15: 1309-1318.

Tollrian, R. 1994. Fish-kairomone induced morphological changes in Daphnia lumholtzi (Sars). Arch. Hydrobiol., 130: 69-75.

Tollrian, R. 1995. Predator-induced morphological defenses: costs, life history shifts, and maternal effects in Daphnia pulex. Ecology, 76: 1691-1705.

Tollrian, R. \& C.D. Harvell. 1999. The ecology and evolution of inducible defenses. Princeton University Press, Princeton: $383 \mathrm{pp}$.

Tollrian, R. \& C. Laforsch. 2006. Linking predator kairomones and turbulence: synergistic effects and ultimate reasons for phenotypic plasticity in Daphnia cucullata. Arch. Hydrobiol., 167: 135-146.

Weber, A. 2001. Interactions between predator kairomone and food level complicate the ecological interpretation of Daphnia laboratory results. J. Plankton Res., 23: 41-46.

Weetman, D. \& D. Atkinson. 2002. Antipredator reaction norms for life history traits in Daphnia pulex: dependence on temperature and food. Oikos, 98: 299-307.

Weber, A. \& S. Declerck. 1997. Phenotypic plasticity of Daphnia life history traits in response to predator kairomones: genetic variability and evolutionary potential. Hydrobiologia, 360: 89-99. 